\title{
Few-mode EDFA Supporting 5 Spatial Modes with Reconfigurable Differential Modal Gain Control
}

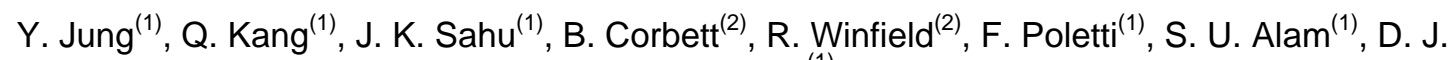 \\ Richardson $^{(1)}$ \\ (1) Optoelectronics Research Centre, University of Southampton, Southampton, SO17 1BJ, UK \\ (2) Tyndall National Institute, University of College Cork, Cork, Ireland \\ ymi@orc.soton.ac.uk
}

\begin{abstract}
We experimentally demonstrate a FM-EDFA supporting 5 spatial modes with reconfigurable differential gain control and excellent gain-flatness obtained through mode-selective bi-directional pumping of a custom fiber with a radially-profiled erbium dopant distribution
\end{abstract}

\section{Introduction}

Mode Division Multiplexing (MDM) whereby selective mode excitation and detection schemes are used to define distinguishable information channels within a few mode fiber (FMF) $[1,2]$ is gathering increasing interest as a cost effective means to accommodate the anticipated future (exponential) growth in internet traffic. Rapid progress has been made in the development of FM transmission fibers and optical amplifiers and a record data transmission capacity of $73.7 \mathrm{~Tb} / \mathrm{s}$ has recently been demonstrated over a $110 \mathrm{~km}$ length link of three-mode fiber (3MF) incorporating an inline 3M-EDFA [2]. A longer transmission distance of $700 \mathrm{~km}$ has been reached at an aggregate linerate of $1.7 \mathrm{~Tb} / \mathrm{s}$ in $3 \mathrm{MF}$ recirculating loop experiments [3]. The maximum reach in this instance was limited by the differential modegroup delay and the propagation of spurious higher order modes (HOMs) within the loop. Although these preliminary demonstrations are encouraging, for MDM to be seriously considered as an alternative to the use of multiple parallel systems based on single-mode, single-core fiber technology, a further increase in the number of parallel transmitted and amplified channels will be necessary.

To this end work has started on higher mode count fibers and a maximum transmission distance of $130 \mathrm{~km}(2 \times 65 \mathrm{~km})$ at a line-rate of $3.8 \mathrm{~Tb} / \mathrm{s}$ over a bandwidth of $400 \mathrm{GHz}$ at $50 \mathrm{GHz}$ channel spacing has been demonstrated using a 6MF [4]. However amplification in this instance was obtained using 6 single mode EDFAs in conjunction with suitable mode demultiplexers due to the lack of a suitable 6M-EDFA. Recently a FM-EDFA supporting 6-spatial modes was reported using two cascaded lengths of FMEDFA with different erbium dopant distributions, resulting in a differential modal gain (DMG) in the region of $4 \mathrm{~dB}$ and with the $\mathrm{LP}_{01}$ mode experiencing the highest gain [5]. It is however well known (and recently demonstrated in Ref [3]) that the HOMs generally experience higher propagation, bending and coupling losses and thus require higher gain for transmission over multiple fiber spans. Mode selective pumping has previously been shown to offer higher gains for the HOMs in a FM-EDFA supporting two mode groups [6], however in this implementation the DMG could not be accurately controlled over the full C-band with a gain flatness of better than $5 \mathrm{~dB}$ and changes by as much as $2.5 \mathrm{~dB}$ depending on the pump power and operating wavelength. Here we report a 5M-EDFA providing adjustable DMG obtained by using a mode-selective bi-directional pumping scheme

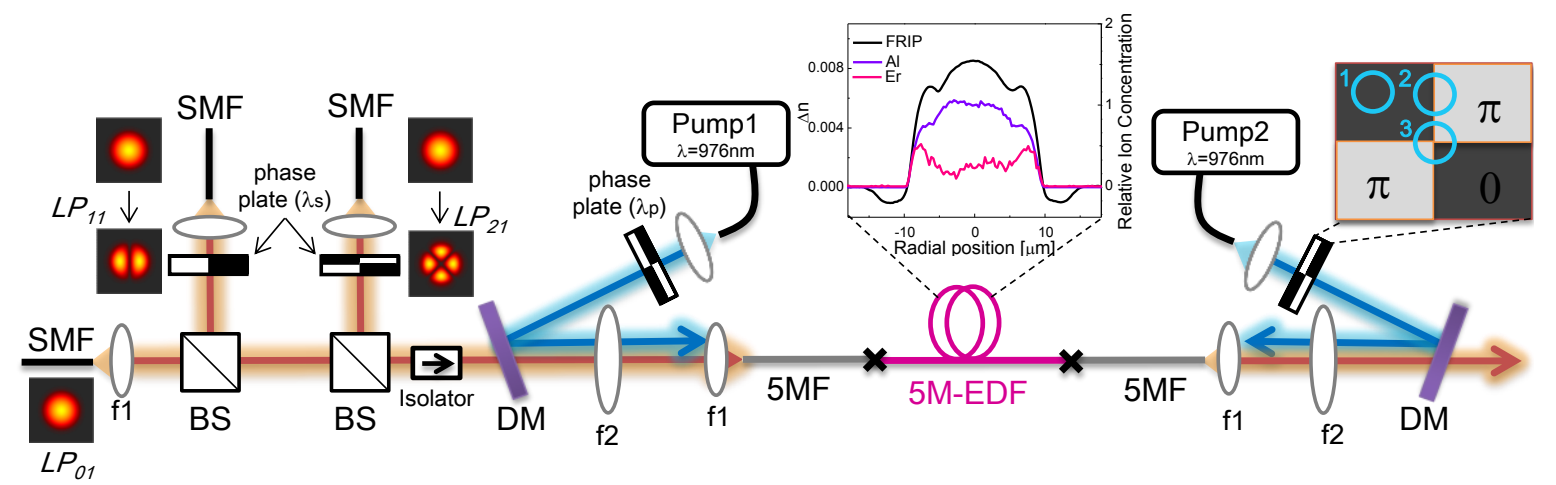

Fig. 1: Schematic diagram of 5-moded erbium doped fiber amplifier (5M-EDFA) gain enhanced by bidirectional pumping configuration. 
in conjunction with an EDF with a specially tailored Er-doping profile. A signal gain of $>20 \mathrm{~dB}$ was obtained for all the guided modes with $D M G$ $<2.5 \mathrm{~dB}$ and excellent gain flatness across the $\mathrm{C}$ band.

\section{Gain performance of 5M-EDFA}

Fig. 1 shows a schematic diagram of our $5 \mathrm{M}$ EDFA for simultaneous amplification of the three lowest spatial mode groups namely $L P_{01}, L_{11}$ and $\mathrm{LP}_{21}$. A mode multiplexer based on phase plates was first used to selectively excite the pure $\mathrm{LP}_{01}, \mathrm{LP}_{11}$ and $\mathrm{LP}_{21}$ signal modes in a passive 5-moded fiber (5MF) with three tunable external cavity lasers. Since the current freespace mode multiplexer scheme suffers from a $3 \mathrm{~dB}$ excess loss for each mode added to the system, the number of spatial modes was limited to three to keep the signal insertion loss within an acceptable level such that the amplifier can be characterized over a wide range of signal input powers. Moreover based on our previous experimental results on 3-mode EDFA [7], we believe that the orthogonal modes of the $\mathrm{LP}_{11}$ and $\mathrm{LP}_{21}$ mode groups will behave similarly. The passive $5 \mathrm{MF}$ was then spliced directly to a $6 \mathrm{~m}$ long ring-doped EDF [8], in which the erbium ions are substantially confined within a ring inside the fiber core to help mitigate the DMG. The 5M-EDF has an outer cladding diameter of $163 \mu \mathrm{m}$ and a core diameter of $18.7 \mu \mathrm{m}$. The estimated effective NA of the core is $\sim 0.14$. The measured absorption at $980 \mathrm{~nm}$ is $8.9 \mathrm{~dB} / \mathrm{m}$. A bidirectional pumping configuration was adopted to provide DMG control. The single mode outputs from two $976 \mathrm{~nm}$ pump laser diodes

(a)

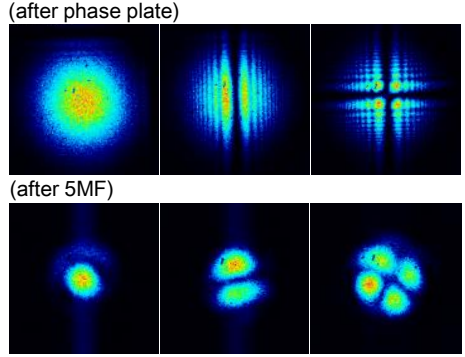

(c)

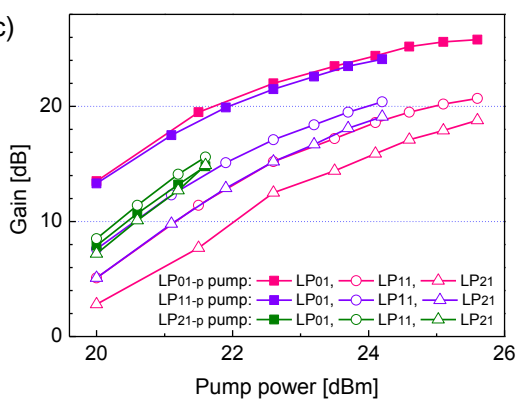

were first converted to the desired pump modes using $980 \mathrm{~nm}$ borosilicate phase plates and then free-space coupled into the two ends of the $5 \mathrm{M}$ EDF. To allow simple but effective modal gain measurement, different wavelengths were chosen for the three different signal channels (for example $1550 \mathrm{~nm}$ for $\mathrm{LP}_{01}, 1555 \mathrm{~nm}$ for $\mathrm{LP}_{11}$ and $1560 \mathrm{~nm}$ for $\mathrm{LP}_{21}$ respectively) and a tunable narrow bandpass filter $(\mathrm{FWHM}=2 \mathrm{~nm})$ and a power meter were used to determine the output power and hence gain of the individual channels, similar to the setup described in Ref. [7]. Splice losses of $0.3 \mathrm{~dB}$ for the $\mathrm{LP}_{01}, 0.8 \mathrm{~dB}$ for the $\mathrm{LP}_{11}$ and $1.0 \mathrm{~dB}$ for the $\mathrm{LP}_{21}$ modes were measured: the variation being due primarily to mode mismatch between the passive $5 \mathrm{MF}$ and active 5M-EDF.

An $\mathrm{LP}_{21}$ pump phase plate was inserted in the optical path of the pump beam to provide reconfigurable pump mode control. Fig. 2a shows the measured CCD images of the spatial profile of the pump mode after the phase plate, when the latter was made to intersect the Gaussian pump beam at different positions, as illustrated by the magnified view of the phase plate in Fig. 1: uniform sector for $\mathrm{LP}_{01}$ excitation (region 1), half-sector for $\mathrm{LP}_{11}$ (region 2) and quadrant-sector for $\mathrm{LP}_{21}$ (region 3 ). The spatial profiles after propagation through a $10 \mathrm{~m}$-long section of passive 5MF are also presented, showing that a clean spatial pump mode was generated by the binary pump phase plate and well-preserved along the fiber in all instances, which is essential in establishing the role of the pump mode profile in mitigation of the DMG of the amplifier. The coupling losses to the 5MF,
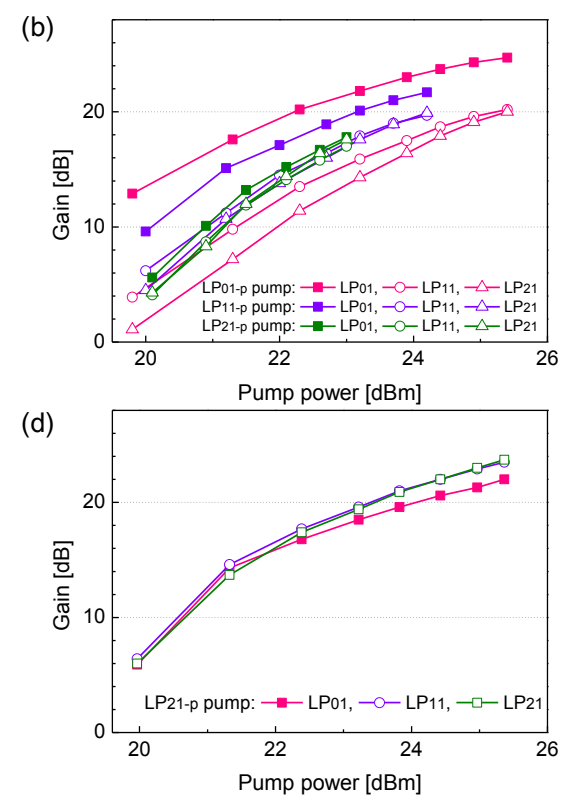

Fig. 2: (a) Pump mode profile after phase plate and after 10m-long 5MF. Mode dependent gain as a function of three different pumping configurations: (b) co-propagating, (c) counter-propagating, and (d) bi-directional. 
however, depend on the particular pump mode and were measured to be $1.1,2.4$ and $3.5 \mathrm{~dB}$ for $\mathrm{LP}_{01-\mathrm{p}}, \quad \mathrm{LP}_{11-\mathrm{p}}$, and $\mathrm{LP}_{21-\mathrm{p}}$ pump modes respectively. These relatively large coupling losses might ultimately be improved by using long period fiber grating assisted mode converter [9] written directly into the pump fiber. Fig. 2 shows the DMG as a function of pump power for different pump spatial modes ( $\mathrm{LP}_{01-\mathrm{p}}$, $\mathrm{LP}_{11-\mathrm{p}}$, and $\mathrm{LP}_{21-\mathrm{p}}$ ) with co-directional (b), counter-directional (c) and bi-directional (d) pumping configurations.

For the co-directional pumping, with an $\mathrm{LP}_{01-\mathrm{p}}$ pump launch condition the $\mathrm{LP}_{01}$ signal experiences much higher gain than the other two modes due to its greater overlap with the erbium ions excited by the $\mathrm{LP}_{01-\mathrm{p}}$ pump. The measured DMG was $7.5 \mathrm{~dB}$ for an input signal power of $-10 \mathrm{dBm}$ and a pump power of $25.5 \mathrm{dBm}$. The DMG decreased to $2.5 \mathrm{~dB}$ when the $\mathrm{LP}_{11-\mathrm{p}}$ pump mode was applied; however the gain for the $\mathrm{LP}_{01}$ signal mode remained higher than that for the other signal modes. For the $\mathrm{LP}_{21-\mathrm{p}}$ pump mode however, the DMG not only dropped below $1 \mathrm{~dB}$ but also the gain for the HOMs became almost equal to that of the fundamental mode. However, as evident from Fig. $2 \mathrm{~b}$, the modal gain was restricted to $17 \mathrm{~dB}$ only for the $\mathrm{LP}_{21-\mathrm{p}}$ pump mode due to the reduced launched pump power caused by the higher coupling losses.

The amplifier behaved similarly for the counterdirectional pumping, as shown in Fig. 2c, albeit with slightly higher modal gains. It is to be noted here that for the same pump power of $21.5 \mathrm{dBm}$, the measured DMG was smaller for the counter pumping configuration, whilst for both pumping configurations there is a clear trend of DMG reduction and relative HOM gain increase relative to the fundamental mode with increasing pump power. Extrapolating these measurements it can be seen that an even higher pump power could lead to power equalization or even to the desirable condition where HOMs experience a larger gain than the fundamental mode. To prove this point, we also tested a bi-directional pump configuration, where an $\mathrm{LP}_{21-p}$ pump mode was injected from both ends with a total coupled pump power of $25.3 \mathrm{dBm}$. As shown in Fig. 2d, maximum modal gains of $22,23.5$, and $23.7 \mathrm{~dB}$ were measured for the $\mathrm{LP}_{01}, \mathrm{LP}_{11}$, and $\mathrm{LP}_{21}$ signal modes, respectively. The modal gain plots show that a wide range of DMGs can be achieved in the various configurations by simply changing the pump power.

Fig. 3a plots the signal gain as a function of input signal power per spatial mode for a fixed launched pump power of $25.3 \mathrm{dBm}$. CCD images
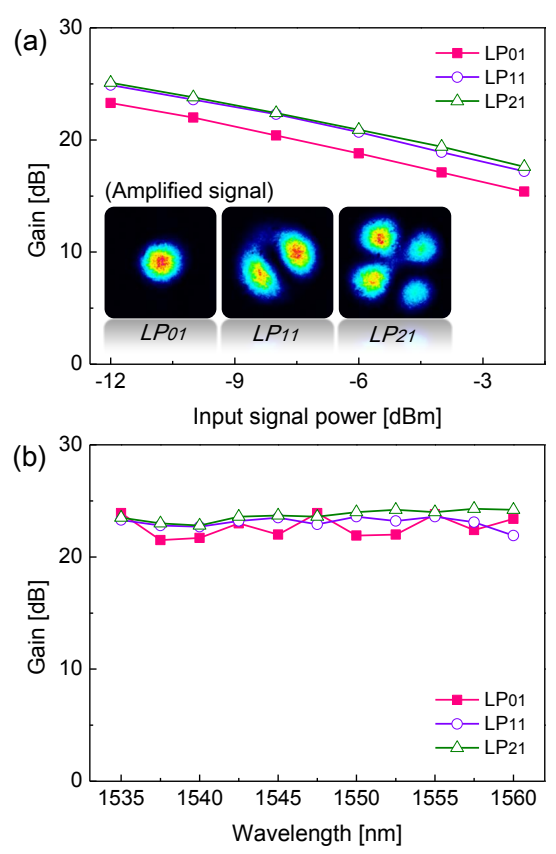

Fig. 3: (a) Measured modal gain as a function of input signal power per mode and (b) gain spectra across the C-band

provide evidence of the clean amplification of the input signals. A maximum aggregate saturated output power of $18.7 \mathrm{dBm}$ was obtained, corresponding to a power conversion efficiency of $21.9 \%$. Fig. $3 b$ plots the measured gain across the C-band for an input signal power of $-10 \mathrm{dBm}$ per mode. The amplifier provides $>20 \mathrm{~dB}$ gain for all three spatial modes with low DMG and a gain flatness of $<2.5 \mathrm{~dB}$ across the C-band.

\section{Conclusions}

We have demonstrated a 5M-EDFA providing reconfigurable mode dependent gain by using a bi-directional $\mathrm{LP}_{21-\mathrm{p}}$ pumping scheme and an Erdoped fiber with a tailored designed ring-doped profile. We consider this an important step in extending MDM transmission both to larger channel numbers and to longer transmission distances and believe the general approach should be further scalable to larger mode numbers.

This work was supported by the European Communities $7^{\text {th }}$ Framework Programme under grant agreement 228033 (MODE-GAP).

\section{References}

[1] S. Randel et al., Opt. Exp. 19, 16697 (2011).

[2] V.A.J.M. Sleiffer, Opt. Exp. 20, B428 (2012).

[3] E. Ip et al., Proc. SPIE 8647, 864709 (2013).

[4] R. Ryf et al., Frontiers in Opt., FW6C (2012).

[5] G.L. Cocq et al., Opt. Exp. 20, 27051 (2012).

[6] N. Bai et al., Opt. Exp. 19, 16601 (2011).

[7] Y. Jung et al., Opt. Exp. 19, B952 (2011).

[8] Y. Jung et al., Opt. Exp. 21, 10383 (2013).

[9] L. Gruner-Nielsen, Sum. Top.,WC1.2 (2012). 